\title{
Etude du rapport coût/sécurite lors de la prise en charge des patients sous antivitamines $\mathrm{K}$ en chirurgie buccale
}

\author{
Survey of the relation cost/safety for patients treated by antivitamines $K$ \\ in oral surgery
}

MATTHIEU CONAN, MURIELLE MASSOT, FABRICE CLIPET, NORA ALNO, SOPHIE LEJEUNE, GILBERT DE MELLO

\section{RÉSUMÉ}

Avant un acte de chirurgie buccale, trois attitudes cliniques sont possibles face aux patients hypocoagulés par antivitamines K (AVK) : maintenir le traitement, l'arrêter ou réaliser un relais héparinique. Quelques mois après la publication par la Société Francophone de Médecine Buccale et de Chirurgie Buccale (SFMBCB) en accord avec la Société Française de Cardiologie (SFC) de recommandations pour la prise en charge de patients sous AVK en chirurgie buccale, cette étude évalue ces recommandations en terme de rapport coût/sécurité. La simulation des coûts globaux de la prise en charge des patients sous AVK pour l'extraction d'une dent incluse réalisée dans ce travail semble montrer une différence entre les attitudes cliniques publiées dans la littérature. II semble plus coûteux de traiter des patients à risque hémorragique important que des patients à risque hémorragique plus faible. Intervenir sans modification du traitement, attitude la plus sûre pour le patient, semble également la moins onéreuse. Lors de la réalisation d'un relais aux héparines, le protocole le plus économique est d'utiliser des héparines de bas poids moléculaire administrées par une infirmière à domicile. L'hospitalisation pour un relais avec des héparines non-fractionnées administrées par voie intraveineuse continue chez le patient à risque thrombotique très important semble être l'attitude la plus onéreuse. L'interruption du traitement AVK expose le patient à des risques d'accidents thromboemboliques potentiellement gravissimes ; cette attitude clinique n'est pas la moins coûteuse.

Ainsi, le rapport coût/sécurité de « l'absence de modification du traitement » est largement positif tandis que celui de «l'interruption du traitement » est largement négatif. (Med Buccale Chir Buccale 2009 ; 15: 17-30).

mots clés: chirurgie buccale, antivitamine $\mathrm{K}$, extraction dentaire, coût, sécurité

\section{SUMMARY}

Dental surgeons have 3 possibilities before a dental extraction when the patient is hypo coagulated by an AVK: stop the treatment, change it by a heparin therapy or change nothing. Risks for the patient are the risk of embolism and the haemorrhagic risk.

This study simulates the different costs and safety of the extraction of an included tooth according to the protocol used for management of the oral anticoagulation treatment.

The first option is very dangerous for the patient because of the extremely serious risk of embolism. This option is not cheaper than the others because of the difficulty to balance the INR after the resumption of the treatment. A lot of biological exams are necessary.

Service de Chirurgie buccale CHU de Rennes Faculté d'Odontologie Université de Rennes 1 Université Européenne de Bretagne

Demande de tirés à part:

Gilbert De Mello Faculté d'Odontologie 2 avenue du Professeur Léon Bernard Bâtiment 1535043 Rennes Cedex

gilbert.de-mello@univ-rennes1.fr 
The second option is expensive because of the biological exams and because of the heparin's injection, at home by a nurse or at hospital. The risk of embolism is also augmented.

The last protocol, to choose if it is possible, is very safe for the patient because there is no higher risk of embolism. Furthermore, this option is very inexpensive.

So, it seems to be better for the cost and the safety of the patient not to change the anticoagulation treatment. (Med Buccale Chir Buccale 2009 ; 15: 17-30).

key words: oral surgery, oral anticoagulant treatment, dental extraction, cost, safety

médecine

buccale

chirurgie

buccale

VOL. $15, \mathrm{~N}^{\circ} 1$ 2009

page 18
Les antivitamines $\mathrm{K}(\mathrm{AVK})$ sont des anticoagulants oraux prescrits pour leur action antithrombotique afin de prévenir les accidents thromboemboliques chez les sujets à risque. Cependant, leur action anticoagulante expose le patient à des risques de saignements difficilement contrôlables lors des actes chirurgicaux. Différentes attitudes cliniques sont parfois possibles en chirurgie buccale pour prévenir ce risque hémorragique et chacune nécessite des moyens humains et matériels différents. L'objectif essentiel de notre étude est de comparer le coût des différentes techniques de prise en charge.

Quelques mois après la publication par la Société Francophone de Médecine Buccale et de Chirurgie Buccale (SFMBCB) en accord avec la Société Française de Cardiologie (SFC) de recommandations pour la prise en charge de patients sous AVK en chirurgie buccale [1], cette étude a donc pour but de traduire ces recommandations en terme de coût pour la santé publique.

\section{MATÉRIEL ET MÉTHODE}

Cette étude a pour but d'analyser les protocoles de prise en charge des patients sous AVK suivants :

1. Sans modification du traitement anticoagulant selon le protocole mis en place dans le service de chirurgie buccale du CHU de Pontchaillou de Rennes.

2. Relais à I'héparine sodique (HNF) administrée par voie intraveineuse avec hospitalisation selon le protocole de Mehra et coll. [2].

3. Relais à l'héparine calcique (HNF) administrée par voie sous-cutanée sans hospitalisation selon le protocole mis en place dans le service de chirurgie buccale du $\mathrm{CHU}$ de Pontchaillou de Rennes.
4. Relais à la dalteparine (HBPM) selon le protocole de Kovacs et coll. [3].

5. Relais à l'enoxaparine (HBPM) selon le protocole réalisé dans le service de chirurgie buccale du CHU de Pontchaillou de Rennes.

6. Interruption du traitement anticoagulant. L'acte de chirurgie buccale choisi est l'extraction d'une dent incluse, côté DC 40 (= 83,6 $€)$.

Ces simulations ont été réalisées sur un patient $A$ à risque thrombotique modéré dont I'INR est stable à 2 et sur un patient $B$ à risque thrombotique important dont l'INR est stable à 3,5. Ces 2 patients sont traités par fluindione (Préviscan ${ }^{\circledR}$ ) uniquement et ne présentent aucune anomalie acquise ou constitutionnelle de l'hémostase.

Les coûts des médicaments, de l'hospitalisation, de la consultation du médecin et du chirurgien ainsi que des visites de l'infirmière qui sont utilisés dans cette étude ont été fournies par le Département Finance du CHU de Rennes et par le Vidal ${ }^{\circledR}$ (Tab. 1). Quant aux examens biologiques, leurs tarifs sont fixés par la Sécurité Sociale et codifiés par la lettre $\mathrm{B}$ : la NFS correspond à B.35; la numération plaquettaire (NP) à B.20 ; le TCA à B. 25 ; I'INR à B.20. À ces cotations, il faut ajouter pour chaque prélèvement sanguin le forfait de sécurité qui correspond à B.4 et le coût du prélèvement à $2,78 €$.

Plusieurs paramètres ont été fixés :

- La quantité de Tissucol ${ }^{\circledR}$ utilisée est de $1 \mathrm{ml}$ lors de la réalisation de l'hémostase de niveau 2.

- En cas de relais héparinique, il n'y a qu'une seule consultation chez le médecin traitant ou spécialiste et il faut 3 jours pour que le traitement AVK soit de nouveau stable chez le patient $A$ et 5 jours chez le patient $B$.

- Les patients $A$ et $B$ pèsent $60 \mathrm{~kg}$.

En accord avec les recommandations de la SFMBCB utilisées dans le service de chirurgie 
Tableau 1 : Tarifs utilisés dans l'étude (Département Finance du CHU de Rennes). Costs used for this study (Financial department of the hospital of Rennes, France).

\begin{tabular}{|c|c|c|}
\hline & & Coût \\
\hline \multirow{6}{*}{ MEDICAMENTS } & \multirow{2}{*}{$\begin{array}{l}\text { Enoxaprarine (Lovenox }{ }^{\circledR} \text {, } \\
0,2 \mathrm{ml}(4000 \mathrm{UI})\end{array}$} & Boîte de 2 seringues $15,73 €$ \\
\hline & & Boîte de 6 seringues $44,03 €$ \\
\hline & \multirow{2}{*}{$\begin{array}{l}\text { Dalteparine }\left(\text { Fragmine }^{\oplus}\right) \\
0,2 \mathrm{ml}(5000 \mathrm{UI})\end{array}$} & Boîte de 2 seringues $13,24 €$ \\
\hline & & Boîte de 6 seringues $37,66 €$ \\
\hline & Héparine sodique (Héparine Choay ${ }^{\circledR}$ ) & 1 flacon de 25000 UI $0,86 €$ \\
\hline & $\begin{array}{l}\left.\text { Héparine calcique (Calciparine }{ }^{\circledR}\right) \\
0,2 \mathrm{ml}(5000 \text { U.I) }\end{array}$ & 1 boîte de 2 seringues $3,33 €$ \\
\hline \multirow{5}{*}{$\begin{array}{c}\text { MOYENS } \\
\text { D'HEMOSTASE LOCALE }\end{array}$} & Vicry ${ }^{\oplus}$ rapide 3.0 & $7,16 €$ \\
\hline & Curaspon ${ }^{\circledast}$ fin & la boîte de 20 éponges $79,74 €$ \\
\hline & Compresses stériles (Stériliux ${ }^{\circledR}$ ) & 1 boîte de $203 €$ \\
\hline & Exacy $\left.\right|^{\oplus}$ ampoules solution buvable & $\begin{array}{l}1 \text { boîte de } 5 \text { ampoules } \\
\text { de } 10 \mathrm{ml} 3,19 €\end{array}$ \\
\hline & Tissucol $^{\circledR}(1 \mathrm{ml})$ & $71,47 €$ \\
\hline \multirow{5}{*}{ EXAMENS BIOLOGIQUES } & Bilan d'hémostase (NFS, TCA, INR) & $26,46 €$ \\
\hline & NFS & $14,31 €$ \\
\hline & Numération plaquettaire & $10,26 €$ \\
\hline & TCA & $11,61 €$ \\
\hline & INR & $10,26 €$ \\
\hline \multirow{2}{*}{ HOSPITALISATION } & Cardiologie & $396 €$ \\
\hline & ORL & $432 €$ \\
\hline INTERVENTION & DC 40 & $83,6 €$ \\
\hline \multirow{3}{*}{ HONORAIRES } & Médecin & $28 €$ \\
\hline & Chirurgien & $21 €$ \\
\hline & Infirmière & Acte + déplacement $4,90 €$ \\
\hline
\end{tabular}

buccale du CHU de Pontchaillou de Rennes, une hémostase locale est réalisée systématiquement que l'on modifie l'anticoagulation orale ou non. Celle-ci comprend l'utilisation d'une éponge de gélatine de Curaspon ${ }^{\circledR}$ fin, d'un fil de suture Vicryl ${ }^{\circledR}$ rapide, de 2 boîtes d'Exacyl ${ }^{\circledR}$ ampoules solution buvable et d'une vingtaine de compresses stériles (Tab. 2).

Au CHU de Rennes, lors de la mise en place d'un relais héparinique avec hospitalisation, le patient est hospitalisé soit dans le service d'oto-rhinolaryngologie, soit en cardiologie. Les coûts indiqués intègrent les dépenses hôtelières ainsi que les dépenses de personnel. II faut également prendre en compte le coût de structure couvrant les dépenses de fonction support (logistique, administration) qui équivaut à $30 \%$ de l'ensemble des charges (dépenses hôtelières + dépense de personnel + dépenses de médicaments + dépenses de laboratoire).

Lors du relais à l'héparine avec administration à domicile, les honoraires de l'infirmière s'élèvent à $2,90 €$ par injection. Cela correspond à l'acte de l'injection, au matériel utilisé (coton, désinfectant) et aux conseils et explications donnés au patient. Il faut ajouter $2 €$ de frais de déplacement. Ainsi, chaque visite d'infirmière pour une injection souscutanée d'héparine correspond à $4,90 €$. médecine

buccale

chirurgie

buccale

VOL. $15, \mathrm{~N}^{\circ} 1$ 2009

page 19 
Tableau 2 : Moyens d'hémostase locale de niveau 1 utilisés dans le service de chirurgie buccale du CHU de Pontchaillou de Rennes.

Cost of materials for local haemostasis (low haemorrhagic risk) used in the oral surgery department of the hospital of Rennes.

\begin{tabular}{|c|c|c|}
\hline Produits & Prix & Total \\
\hline Curaspon ${ }^{\circledR}$ fin (1 éponge) & $79,74 / 20=4 €$ & \\
\hline Vicryl ${ }^{\circledR}$ rapide (1 boîte) & $7,16 €$ & \\
\hline $\begin{array}{l}\text { Exacyl }^{\circledR} \text { ampoules solution buvable } \\
\text { ( } 2 \text { boîtes de } 5 \text { ) }\end{array}$ & $6,38 €$ & $20,50 €$ \\
\hline Compresses stériles Stérilux ${ }^{\circledast}$ ( 1 boîte de 20 ) & $3 €$ & \\
\hline
\end{tabular}

\section{RÉSULTATS}

médecine

buccale

chirurgie

buccale

VOL. $15, \mathrm{~N}^{\circ} 1$ 2009

page 20

\section{Sans modification du traitement AVK}

Selon les recommandations de la SFMBCB et de la SFC, une mesure de l'INR doit être systématiquement réalisée avant la prise en charge des patients sous AVK en chirurgie buccale. Une hémostase locale de niveau 2 est effectuée pour le patient $B$. Dans le service de chirurgie buccale du $\mathrm{CHU}$ de Pontchaillou de Rennes, celle-ci est réalisée en utilisant les produits de l'hémostase locale de niveau 1 et $1 \mathrm{ml}$ de colle biologique $\left(\right.$ Tissucol $\left.{ }^{\circledR}\right)$. Aucun des patients n'est hospitalisé (Tab. 3).

Tableau 3 : Coût de l'extraction d'une dent incluse sans modification du traitement AVK selon le protocole du service de chirurgie buccale du CHU de Pontchaillou de Rennes.

Cost of an included tooth's extraction, without any modification of AVK treatment, like in the oral surgery department of the hospital of Rennes.

\begin{tabular}{|c|c|c|}
\hline Patient & Prestations & Total \\
\hline A & $\begin{array}{c}\text { Consultation du praticien } \\
\qquad 1 \text { INR } \\
\text { Acte chirurgical } \\
\text { Hémostase locale de niveau } 1\end{array}$ & $135 €$ \\
\hline B & $\begin{array}{l}\text { Consultation du praticien } \\
\qquad 1 \mathrm{INR} \\
\text { Acte chirurgical } \\
\text { Hémostase locale de niveau } 1 \\
\text { Tissucol }^{\circledR}(1 \mathrm{ml})\end{array}$ & $210 €$ \\
\hline
\end{tabular}




\section{Relais aux héparines non fractionnées}

Pour le calcul, nous avons utilisé 2 protocoles différents : celui décrit par Mehra et coll. [2] avec hospitalisation du patient et administration de I'héparine sodique par voie intraveineuse en perfusion continue, et celui appliqué dans le service de chirurgie buccale du $\mathrm{CHU}$ de Pontchaillou de Rennes sans hospitalisation du patient et avec administration de Calciparine ${ }^{\circledR}$ par voie souscutanée.

Selon le protocole de Mehra et coll. [2], 2 jours avant d'hospitaliser le patient, on effectue un bilan d'hémostase et l'anticoagulation orale est interrompue. Le patient est hospitalisé 3 jours avant l'intervention et on lui injecte l'héparine sodique $80 \mathrm{Ul} / \mathrm{kg}$ en bolus, puis une perfusion de $18 \mathrm{UI} / \mathrm{kg} / \mathrm{h}$ pendant 6 heures. On contrôle le TCA 6 heures après le bolus afin d'ajuster la posologie de la perfusion. Selon le protocole de Mehra et coll. [2], la perfusion minimale administrable est donnée au patient $A$, c'est-à-dire $15 \mathrm{UI} / \mathrm{kg} / \mathrm{h}$. La perfusion maximale administrable est donnée au patient $\mathrm{B}$, soit un $2^{\mathrm{e}}$ bolus de $80 \mathrm{UI} / \mathrm{kg}$, puis une perfusion de $22 \mathrm{UI} / \mathrm{kg} / \mathrm{h}$. Cette perfusion est interrompue 4 heures avant l'intervention et reprise immédiatement après à la même posologie jusqu'au $3^{\mathrm{e}}$ jour postopératoire pour le patient $A$ et jusqu'au $5^{\mathrm{e}}$ jour postopératoire pour le patient $B$. La dose totale d'héparine sodique administrée au patient A est donc de $215000 \mathrm{U} . \mathrm{I}$ et de 400000 U.I pour le patient B. Le TCA est contrôlé toutes les 6 heures après chaque changement de posologie de la perfusion jusqu'à ce que 2 TCA successifs soient en zone thérapeutique, puis toutes les 24 heures. Ainsi, pour le patient A, 3 TCA sont réalisés lors de la mise en route du relais 3 jours avant l'intervention puis un à $\mathrm{J}-2$, à $\mathrm{J}-1$ et à $\mathrm{J} 0$, ce qui fait un nombre total de 6 TCA. Pour le patient $B, 5$ TCA sont réalisés au début du relais à $\mathrm{J}-3$ puis un à $\mathrm{J}-2$, à $\mathrm{J}-1$ et un à $\mathrm{J} 0$, ce qui fait un total de 8 TCA. La NP est contrôlée tous les 3 jours pendant toute la durée du relais, ce qui fait un nombre total de 2 NP pour le patient A et 3 NP pour le patient B. Selon Mehra et coll. [2], afin de contrôler la reprise de l'anticoagulation orale, un INR est prescrit le lendemain et le surlendemain de l'intervention et le jour de la sortie de l'hôpital du patient. Ainsi, le nombre total d'INR réalisés pour le patient $A$ et pour le patient $B$ est de 3. Le patient $A$ est donc hospitalisé 7 jours et le patient B 9 jours. Au CHU de Rennes, ceux-ci peuvent être hospitalisés soit dans le service de cardiologie soit dans le service d'ORL. Avant la mise en place de ce relais, une consultation d'anesthésie est bien entendu obligatoire.

Plusieurs paramètres sont à intégrer pour le calcul des frais d'hospitalisation. Le coût de la journée d'hospitalisation intègre les dépenses hôtelières et les frais de personnel. Les dépenses médicales intègrent la consultation du médecin anesthésiste et du praticien qui va réaliser l'acte chirurgical, le prix de l'intervention, l'héparine sodique administrée au patient et les moyens d'hémostase locale utilisés. II faut intégrer les prestations consommées qui sont ici les examens de laboratoire. Enfin, il faut prendre en compte le coût de structure couvrant les fonctions supports telles que la logistique et l'administration ; il correspond à $30 \%$ de l'ensemble des charges précédentes (Tab. 4).

Ainsi, l'ensemble du coût de l'hospitalisation, des dépenses médicales et des prestations consommées est, pour le patient $A$ de $3050 €$ s'il est hospitalisé en cardiologie et de $3300 €$ s'il est hospitalisé en ORL et pour le patient B de 3 $900 €$ s'il est hospitalisé en cardiologie et de 4 $200 €$ s'il est hospitalisé en ORL. Lorsqu'on intègre le coût de structure, le coût global est donc compris entre $4000 €$ et $4300 €$ pour le patient $A$ et entre $5000 €$ et $5500 €$ pour le patient B (Tab. 5).

Selon le protocole mis en place dans le service de chirurgie buccale du CHU de Pontchaillou de Rennes, l'administration de l'héparine non-fractionnée (Calciparine ${ }^{\circledR}$ ) se fait par une infirmière à domicile par des injections sous-cutanées 2 à 3 fois par jour selon que le patient est à risque d'accident thromboembolique modéré ou important.

Pour le patient $A$, un bilan d'hémostase est réalisé et il arrête son traitement AVK 4 jours avant l'intervention s'il s'agit de fluindione (Préviscan ${ }^{\circledR}$ ). Les administrations de Calciparine ${ }^{\circledR}$ débutent le lendemain avec une injection le matin et une injection le soir de $0,2 \mathrm{ml}$ (5 $000 \mathrm{UI})$ chacune. On contrôle l'INR à J-2 et la NP et le TCA la veille ou le jour de l'intervention. La dernière injection de médecine

buccale

chirurgie

buccale

VOL. $15, \mathrm{~N}^{\circ} 1$ 2009

page 21 
Tableau 4 : Frais d'hospitalisation.

Cost of a hospitalisation.

\begin{tabular}{|c|c|c|}
\hline Journée d'hospitalisation & En ORL & $432 €$ \\
\hline \multirow{2}{*}{ Dépenses médicales } & Consultation du médecin anesthésiste & $396 €$ \\
\hline Consultation du chirurgien & $28 €$ \\
\hline Prestations consommées & Acte chirurgical & $83,6 €$ \\
& Héparine sodique & 1 flacon de 25000 UI \\
\hline Hémostase locale de niveau 1 & $0,86 €$ \\
\hline Structure & Bilan d'hémostase & $20,50 €$ \\
\hline INR & $26,46 €$ \\
\hline
\end{tabular}

médecine buccale chirurgie buccale

VOL. $15, \mathrm{~N}^{\circ} 1$ 2009 page 22

Tableau 5 : Coût de l'extraction d'une dent incluse avec mise en place d'un relais à l'héparine sodique administrée par voie intraveineuse selon le protocole de Mehra et coll. [2].

Cost of an included tooth's extraction, with modification of the AVK treatment by a heparin therapy (Mehra's protocol).

\begin{tabular}{c|c|c}
\hline Patients & Services dans lequel le patient est hospitalisé & Coût global \\
\hline A & Cardiologie & $4000 €$ \\
\hline B & Oto-rhino-laryngologie & $4300 €$ \\
\hline
\end{tabular}

Calciparine ${ }^{\circledR}$ a lieu 12 heures avant l'intervention. Le traitement anticoagulant oral ainsi que les injections d'héparine sont repris 8 heures après l'intervention, un INR et une NP sont réalisés le surlendemain de l'intervention. Le troisième jour après l'intervention, on contrôle à nouveau l'INR et les injections de Calciparine ${ }^{\circledR}$ sont arrêtées.

Ainsi, le nombre total d'injections d'héparine de $0,2 \mathrm{ml}(5000 \mathrm{U} . \mathrm{l})$ est de 13 , ce qui correspond à $2,6 \mathrm{ml}$ (65000 UI) de Calciparine ${ }^{\circledR}$. II y a donc 13 visites de l'infirmière, une consultation du médecin anesthésiste ou du cardiologue, une consultation du praticien qui va réaliser l'extraction, un bilan d'hémostase, 2 NP, 3 INR et un TCA. II faut compter également le prix de l'intervention ainsi que le coût de l'hémostase locale de niveau 1 réalisée (Tab. 6).

En ce qui concerne le patient $B$, un bilan d'hémostase est préalablement réalisé et le patient arrête son traitement anticoagulant oral 4 jours avant l'intervention. L'administration de Calciparine ${ }^{\circledR}$ débute le lendemain par 3 injections par jour de $0,2 \mathrm{ml}$ (5 $000 \mathrm{UI})$ chacune. On contrôle I'INR à J-2 et la NP et le TCA la veille ou le jour de l'intervention. La dernière injection se fait 8 heures avant l'intervention. Le jour de l'intervention, l'injection du matin n'est pas réalisée. Les injections d'HNF et l'administration d'AVK reprennent 68 heures après l'intervention à la posologie préopératoire. $A \mathrm{~J}+2$, un INR et une NP sont 
effectués. $A \mathrm{~J}+4$, on contrôle l'INR et à $\mathrm{J}+5$, on réalise une NP et un INR afin de s'assurer que celui-ci est stable à un niveau thérapeutique. L'administration de Calciparine ${ }^{\circledR}$ est alors arrêtée. Ainsi, le nombre total d'injections d'héparine de $0,2 \mathrm{ml}(5000 \mathrm{UI})$ est de 26 , ce qui correspond à $5,2 \mathrm{ml}(130000 \mathrm{UI})$ de Calciparine ${ }^{\circledR}$. II y a donc 26 visites de l'infirmière, une consultation du médecin anesthésiste ou du cardiologue, une consultation du praticien qui va réaliser l'extraction, un bilan d'hémostase, 3 NP, 4 INR et 1 TCA. Il faut compter à nouveau le prix de l'intervention ainsi que le coût de l'hémostase locale de niveau 1 réalisée (Tab. 6).

\section{Relais avec des héparines de bas poids moléculaire}

Pour le calcul, 2 protocoles différents ont été utilisés : celui décrit dans la littérature par Kovacs et coll. [3] avec administration sous-cutanée de dalteparine (Fragmine ${ }^{\circledR}$ ) et celui mis en place dans le service de chirurgie buccale du CHU de Pontchaillou de Rennes avec administration souscutanée d'enoxaparine $\left(\right.$ Lovenox $\left.^{\circledR}\right)$. Le patient n'est pas hospitalisé et les injections sont réalisées par une infirmière à domicile.

Selon le protocole de Kovacs et coll. [3], pour le patient $A$, après avoir réalisé un bilan d'hémostase, la fluindione est interrompue 4 jours avant

Tableau 6 : Calcul du coût de l'extraction d'une dent incluse avec relais à la Calciparine ${ }^{\circledR}$ en suivant le protocole mis en place dans le Service de chirurgie buccale du CHU de Pontchaillou de Rennes.

Cost of an included tooth's extraction, with modification of the AVK treatment by a Calciparine ${ }^{\circledR}$ therapy (protocol used in the oral surgery department of the hospital of Rennes).

\begin{tabular}{|c|c|c|c|}
\hline Patient & Paramètres à intégrer & Coût & Total \\
\hline A & $\begin{array}{c}\text { Calciparine }{ }^{\circledR} \\
\text { Honoraires de l'infirmière } \\
1 \text { consultation du médecin } \\
1 \text { consultation du praticien } \\
\text { Acte chirurgical } \\
\text { Hémostase locale } \\
\text { de niveau } 1 \\
1 \text { bilan d'hémostase } \\
2 \text { NP } \\
3 \text { INR } \\
1 \text { TCA }\end{array}$ & $\begin{array}{c}13 \times(3,33 / 2) € \\
13 \times 4,90 € \\
28 € \\
21 € \\
83,60 € \\
20,50 € \\
26,46 € \\
2 \times 10,26 € \\
3 \times 10,26 € \\
11,61 €\end{array}$ & $330 €$ \\
\hline B & $\begin{array}{c}\text { Calciparine }{ }^{\circledR} \\
\text { Honoraires de l'infirmière } \\
1 \text { consultation du médecin } \\
1 \text { consultation du praticien } \\
\text { Acte chirurgical } \\
\text { Hémostase locale } \\
\text { de niveau } 1 \\
1 \text { bilan d'hémostase } \\
3 \text { NP } \\
4 \text { INR } \\
1 \text { TCA }\end{array}$ & $\begin{array}{c}26 \times(3,33 / 2) € \\
26 \times 4,90 € \\
28 € \\
21 € \\
83,60 € \\
20,50 € \\
26,46 € \\
3 \times 10,26 € \\
4 \times 10,26 € \\
11,61 €\end{array}$ & $435 €$ \\
\hline
\end{tabular}

médecine buccale chirurgie buccale

VOL. $15, \mathrm{~N}^{\circ} 1$ 2009

page 23 
médecine buccale chirurgie buccale

VOL. $15, \mathrm{~N}^{\circ} 1$ 2009

page 24 l'intervention. On débute le lendemain les injections biquotidiennes de Fragmine ${ }^{\circledR}$ de $100 \mathrm{UI} / \mathrm{kg}$ chacune. La veille de l'intervention, seule l'injection de Fragmine ${ }^{\circledR}$ du matin est réalisée et l'on contrôle la NP et l'INR. II n'y a aucune injection de Fragmine ${ }^{\circledR}$ le jour de l'intervention et les AVK sont repris dès le soir de l'intervention. L'administration biquotidienne de Fragmine ${ }^{\circledR}$ reprend le lendemain. On contrôle la reprise de l'anticoagulation orale avec un INR prescrit à J+2. Trois jours après l'intervention, on contrôle à nouveau la NP et l'INR du patient afin de s'assurer que le traitement anticoagulant est de nouveau stabilisé à un niveau thérapeutique. Ainsi, le nombre total d'injections de Fragmine ${ }^{\circledR}$ de $0,2 \mathrm{ml}$ (5000 UI) est 11, ce qui correspond environ à $2,2 \mathrm{ml}$ et à $55000 \mathrm{UI}$ de dalteparine. II y a donc 11 visites de l'infirmière, une consultation du médecin anesthésiste ou du cardiologue, une consultation du praticien qui va réaliser l'extraction, un bilan d'hémostase, 2 NP, 3 INR et un TCA. Il faut compter également le prix de l'intervention ainsi que le coût de l'hémostase locale de niveau 1 réalisée (Tab. 7).

Tableau 7 : Calcul du coût de l'extraction d'une dent incluse avec relais à la Fragmine ${ }^{\circledR}$ en suivant le protocole de Kovacs et coll. ${ }^{[3]}$.

Cost of an included tooth's extraction, with modification of the AVK treatment by a Fragmine ${ }^{\circledR}$ therapy (Kovacs's protocol).

\begin{tabular}{|c|c|c|c|}
\hline Patients & Paramètres à intégrer & Coût & Total \\
\hline A & $\begin{array}{c}\text { Fragmine }^{\oplus} \\
\text { Honoraires de l'infirmière } \\
1 \text { consultation } \\
\text { du médecin } \\
1 \text { consultation } \\
\text { du praticien } \\
\text { Acte chirurgical } \\
\text { Hémostase locale } \\
\text { de niveau } 1 \\
1 \text { bilan d'hémostase } \\
2 \text { NP } \\
3 \text { INR } \\
1 \text { TCA }\end{array}$ & $\begin{array}{c}11 \times(13,24 / 2) € \\
11 \times 4,90 € \\
28 € \\
21 € \\
83,60 € \\
20,50 € \\
26,46 € \\
2 \times 10,26 € \\
3 \times 10,26 € \\
11,61 €\end{array}$ & $370 €$ \\
\hline B & $\begin{array}{c}\text { Fragmine }^{\oplus} \\
\text { Honoraires de l'infirmière } \\
1 \text { consultation } \\
\text { du médecin } \\
1 \text { consultation } \\
\text { du praticien } \\
\text { Acte chirurgical } \\
\text { Hémostase locale } \\
\text { de niveau 1 } \\
1 \text { bilan d'hémostase } \\
2 \text { NP } \\
4 \text { INR } \\
1 \text { TCA }\end{array}$ & $\begin{array}{c}15 \times(13,24 / 2) € \\
15 \times 4,90 € \\
28 € \\
21 € \\
83,60 € \\
20,50 € \\
26,46 € \\
2 \times 10,26 € \\
4 \times 10,26 € \\
11,61 €\end{array}$ & $425 €$ \\
\hline
\end{tabular}


Pour le patient $B$, un bilan d'hémostase est également réalisé. Quatre jours avant l'intervention, la fluindione est arrêtée. On débute les injections de Fragmine ${ }^{\circledR}, 100 \mathrm{UI} / \mathrm{kg}$ matin et soir le lendemain. La veille de l'intervention, on ne réalise que l'injection du matin de Fragmine ${ }^{\circledR}$ et une mesure de la NP et de l'INR est effectuée. Le jour de l'intervention, on contrôle à nouveau l'INR et aucune injection de Fragmine ${ }^{\circledR}$ n'est réalisée. Le traitement anticoagulant oral est repris dès le soir de l'intervention et les injections d'héparine sont reprises le lendemain. Une NP est effectuée à $J+4$ et l'INR est contrôlé à $\mathrm{J}+4$ et $\mathrm{J}+5$ afin de s'assurer que le traitement $\mathrm{AVK}$ est stable à un niveau thérapeutique. Les injections de Fragmine ${ }^{\circledR}$ sont alors interrompues.

Ainsi, le nombre total d'injections de Fragmine ${ }^{\circledR}$ de $0,2 \mathrm{ml}$ (5000 UI) est de 15, ce qui correspond environ à $3 \mathrm{ml}$ et à $75000 \mathrm{UI}$ de dalteparine. II y a donc 15 visites de l'infirmière, une consultation du médecin anesthésiste ou du cardiologue, une consultation du praticien qui va réaliser l'extraction, un bilan d'hémostase, 2 NP, 4 INR et un TCA. II faut compter également le prix de l'intervention ainsi que le coût de l'hémostase locale de niveau 1 réalisée (Tab. 7 ).

Selon le protocole mis en place dans le service de chirurgie buccale du CHU de Pontchaillou de Rennes, le relais aux HBPM se fait avec de l'enoxaparine (Lovenox ${ }^{\circledR}$ ).

Pour le patient $A$, un bilan d'hémostase est effectué et la fluindione est arrêtée 4 jours avant l'intervention. L'administration d'enoxaparine par voie sous-cutané débute le lendemain à la posologie d'une injection de 0,4 ml (4 $000 \mathrm{UI}$ ) par jour. On contrôle la NP 2 jours avant l'intervention. II n'y a pas d'injection d'enoxaparine le matin de l'intervention. Elles sont reprises, ainsi que l'anticoagulation orale, 12 heures après la l'intervention. L'INR est contrôlé le surlendemain de l'intervention et à $\mathrm{J}+3$ afin de s'assurer qu'il est stable à un niveau thérapeutique. L'administration de Lovenox ${ }^{\circledR}$ est alors interrompue. Ainsi, le nombre total d'injections de Lovenox ${ }^{\circledR}$ de $0,4 \mathrm{ml}$ (4 $000 \mathrm{UI})$ est de 7 , ce qui correspond environ à $2,8 \mathrm{ml}$ et à $28000 \mathrm{Ul}$ d'enoxaparine. II y a donc 7 visites de l'infirmière, une consultation du médecin anesthésiste ou du cardiologue, une consultation du praticien qui va réaliser l'extraction, un bilan d'hémostase, 2 NP et 2 INR. II faut compter également le prix de l'intervention ainsi que le coût de l'hémostase locale de niveau 1 réalisée (Tab. 8).

Pour le patient $B$, le bilan d'hémostase est effectué, la fluindione interrompue 4 jours avant l'intervention et l'administration d'enoxaparine débute le lendemain par une injection le matin et une injection le soir de 0,4 ml (4 $000 \mathrm{UI})$ chacune. La NP est contrôlée le lendemain du début des injections. Une seule injection d'enoxaparine est réalisée la veille de l'intervention. II n'y a pas d'administration de Lovenox ${ }^{\circledR}$ le matin de l'intervention. Elle est reprise, ainsi que la fluindione le soir de l'intervention. On contrôle l'INR à J+2, J+4 et $\mathrm{J}+5$ afin de s'assurer de la bonne reprise de l'anticoagulation orale et la NP à $\mathrm{J}+3$. Les injections d'héparine sont interrompues 5 jours après l'intervention quand l'anticoagulation orale est de nouveau stable à un niveau thérapeutique.

Ainsi, le nombre total d'injections de Lovenox ${ }^{\circledR}$ de $0,4 \mathrm{ml}$ (4 $000 \mathrm{UI}$ ) est 16, ce qui correspond environ à 6,4 $\mathrm{ml}$ et à $64000 \mathrm{UI}$ d'enoxaparine. II y a donc 16 visites de l'infirmière, une consultation du médecin anesthésiste ou du cardiologue, une consultation du praticien qui va réaliser l'extraction, un bilan d'hémostase, 2 NP et 3 INR. II faut compter également le prix de l'intervention ainsi que le coût de l'hémostase locale de niveau 1 réalisée (Tabl. 8).

\section{Interruption du traitement AVK}

Selon les recommandations de prise en charge de patients sous AVK en chirurgie buccale, cette attitude doit restée exceptionnelle et réservée à des patients à faible risque d'accident thromboembolique. Elle est décidée par le médecin traitant ou le médecin spécialiste. II n'y a pas d'hospitalisation du patient et une hémostase locale de niveau 1 est réalisée. II n'y a pas de protocole clairement décrit dans la littérature et, pour le calcul, nous nous sommes appuyés sur plusieurs articles ${ }^{[4,5,6]}$ qui recommandent en moyenne un contrôle de l'INR tous les 2 jours.

Pour le patient $A$, un bilan d'hémostase est effectué et la fluindione est interrompue 4 jours avant I'intervention. On contrôle l'INR à J-2, J0, J+2 et médecine

buccale

chirurgie

buccale

VOL. $15, \mathrm{~N}^{\circ} 1$ 2009

page 25 
médecine

buccale

chirurgie

buccale

VOL. $15, \mathrm{~N}^{\circ} 1$ 2009

page 26

Tableau 8: Calcul du coût de l'extraction d'une dent incluse avec relais au Lovenox ${ }^{\circledR}$ en suivant le protocole mis en place dans le service de chirurgie buccale du CHU de Pontchaillou de Rennes.

Cost of an included tooth's extraction, with modification of the AVK treatment by a Lovenox ${ }^{\circledR}$ therapy (protocol used in the oral surgery department of the hospital of Rennes).

\begin{tabular}{|c|c|c|c|}
\hline Patients & Paramètres à intégrer & Coût & Total \\
\hline$A$ & $\begin{array}{c}\text { Lovenox }^{\oplus} \\
\text { Honoraires de l'infirmière } \\
1 \text { consultation du médecin } \\
1 \text { consultation du praticien } \\
\text { Acte chirurgical } \\
\text { Hémostase locale de niveau } 1 \\
1 \text { bilan d'hémostase } \\
2 \text { NP } \\
2 \text { INR }\end{array}$ & $\begin{array}{c}7 \times(15,73 / 2) € \\
7 \times 4,90 € \\
28 € \\
21 € \\
83,60 € \\
20,50 € \\
26,46 € \\
2 \times 10,26 € \\
2 \times 10,26 €\end{array}$ & $310 €$ \\
\hline B & $\begin{array}{c}\text { Lovenox }^{\circledast} \\
\text { Honoraires de l'infirmière } \\
1 \text { consultation du médecin } \\
1 \text { consultation du praticien } \\
\text { Acte chirurgical } \\
\text { Hémostase locale de niveau } 1 \\
1 \text { bilan d'hémostase } \\
2 \text { NP } \\
3 \text { INR }\end{array}$ & $\begin{array}{c}16 \times(15,73 / 2) € \\
16 \times 4,90 € \\
28 € \\
21 € \\
83,60 € \\
20,50 € \\
26,46 € \\
2 \times 10,26 € \\
3 \times 10,26 €\end{array}$ & $425 €$ \\
\hline
\end{tabular}

J+4. Le traitement AVK est repris le soir de l'intervention.

Pour le patient $B$, le bilan d'hémostase est réalisé et la fluindione est interrompue 5 jours avant l'intervention. On contrôle l'INR à J-2, J0, J+2, J+4 et $\mathrm{J}+5$. Les AVK est repris le soir de l'intervention (Tab. 9).

\section{DISCUSSION}

Cette simulation montre une différence de coût global de l'extraction d'une dent incluse chez un patient sous AVK selon l'attitude thérapeutique (Tab. 10, Fig. 1 et 2).

Ainsi, différentes remarques peuvent être faites à partir de cette simulation. En effet, il semble que : - la prise en charge d'un patient à risque hémorragique important est plus coûteuse que la prise en charge d'un patient à risque hémorragique modéré;

- l'attitude la moins coûteuse est d'intervenir sans modifier l'anticoagulation orale ;

- lors de la réalisation d'un relais héparinique, l'utilisation d'HBPM est moins onéreuse que l'utilisation d'HNF ; 
Tableau 9 : Calcul du coût, pour les patients A et B, de l'extraction d'une dent incluse avec interruption du traitement anticoagulant.

Cost of an included tooth's extraction, with stopping of the AVK treatment.

\begin{tabular}{|c|c|c|c|}
\hline Patient & Paramètres à intégrer & Coût & Total \\
\hline A & $\begin{array}{c}1 \text { consultation du médecin } \\
1 \text { consultation du praticien } \\
\text { Acte chirurgical } \\
\text { Hémostase locale de niveau } 1 \\
1 \text { bilan d'hémostase } \\
4 \text { INR }\end{array}$ & $\begin{array}{c}28 € \\
21 € \\
83,60 € \\
20,50 € \\
26,46 € \\
4 \times 10,26 €\end{array}$ & $220 €$ \\
\hline B & $\begin{array}{c}1 \text { consultation du médecin } \\
1 \text { consultation du praticien } \\
\text { Acte chirurgical } \\
\text { Hémostase locale de niveau } 1 \\
1 \text { bilan d'hémostase } \\
5 \text { INR }\end{array}$ & $\begin{array}{c}28 € \\
21 € \\
83,60 € \\
20,50 € \\
26,46 € \\
5 \times 10,26 €\end{array}$ & $230 €$ \\
\hline
\end{tabular}

Tableau 10 : Tableau récapitulatif du coût global de l'extraction d'une dent incluse chez un patient traité par AVK à risque hémorragique modéré (patient $A$ ) et à risque hémorragique important (patient $B$ ) selon les différentes attitudes de prise en charge.

Cost of an included tooth's extraction according to the used protocol.

\begin{tabular}{|c|c|c|}
\hline Techniques de prise en charge & Patient A & Patient B \\
\hline Sans modification du traitement AVK & $135 €$ & $210 €$ \\
\hline $\begin{array}{l}\text { Relais avec des HNF avec hospitalisation selon le } \\
\text { protocole de Mehra et coll. }\end{array}$ & $4300 €$ & $5500 €$ \\
\hline $\begin{array}{l}\text { Relais avec des HNF sans hospitalisation selon le } \\
\text { protocole mis en place au CHU de Rennes }\end{array}$ & $330 €$ & $435 €$ \\
\hline $\begin{array}{l}\text { Relais avec des HBPM selon le protocole de Kovacs } \\
\text { et coll. }\end{array}$ & $370 €$ & $425 €$ \\
\hline $\begin{array}{l}\text { Relais avec des HBPM selon le protocole mis en } \\
\text { place au CHU de Rennes }\end{array}$ & $310 €$ & $425 €$ \\
\hline Interruption du traitement AVK & $220 €$ & $230 €$ \\
\hline
\end{tabular}

\section{buccale \\ chirurgie \\ buccale}

médecin

VOL. $15, \mathrm{~N}^{\circ} 1$

2009

page 27 


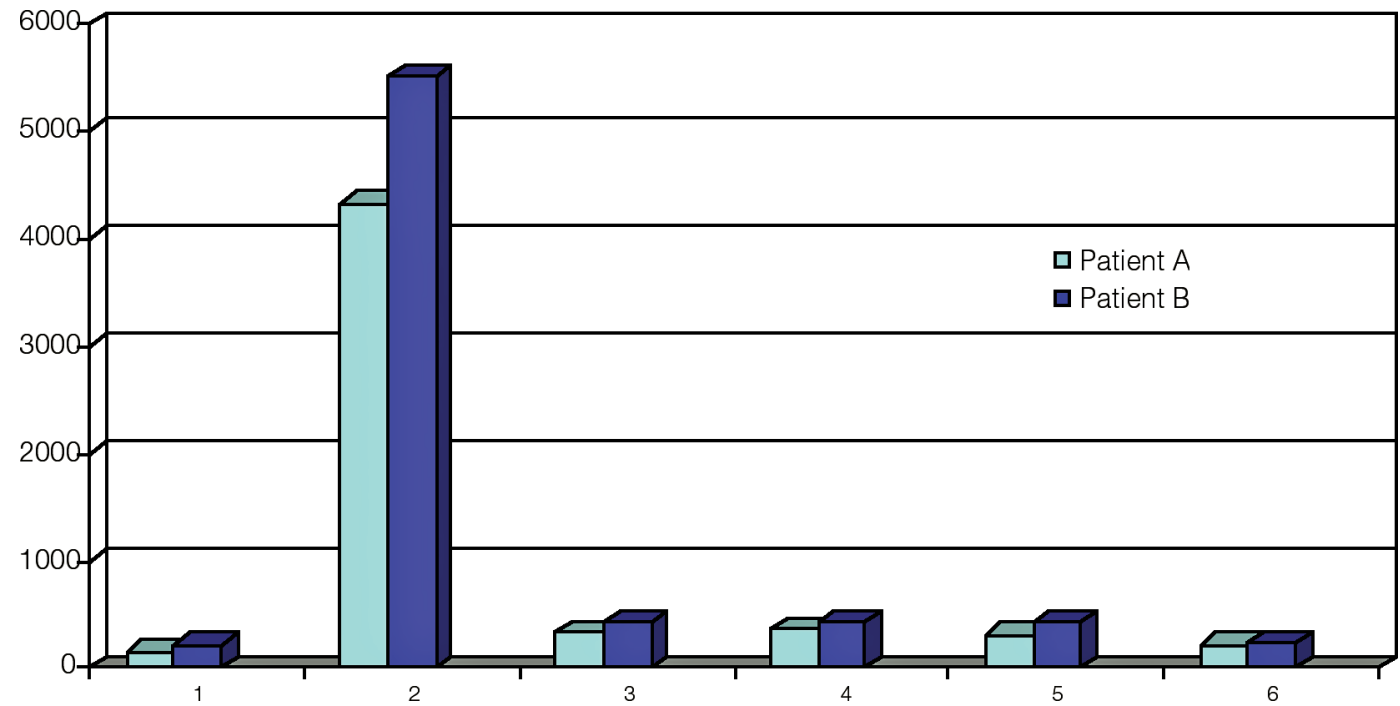

Figure 1 : Coûts globaux des différents modes de prise en charge du patient sous AVK pour l'extraction d'une dent incluse. Cost of an included tooth's extraction according to the used protocol.

1. Sans modification du traitement AVK

2. Relais avec des HNF et hospitalisation selon le protocole de Mehra et coll.

médecine

buccale

chirurgie

buccale

VOL. $15, \mathrm{~N}^{\circ} 1$ 2009

page 28

3. Relais avec des HNF sans hospitalisation selon le protocole de Pontchaillou (Rennes)

4. Relais avec des HBPM selon le protocole de Kovacs et coll.

5. Relais avec des HBPM selon le protocole de Pontchaillou (Rennes)

6. Interruption du traitement AVK

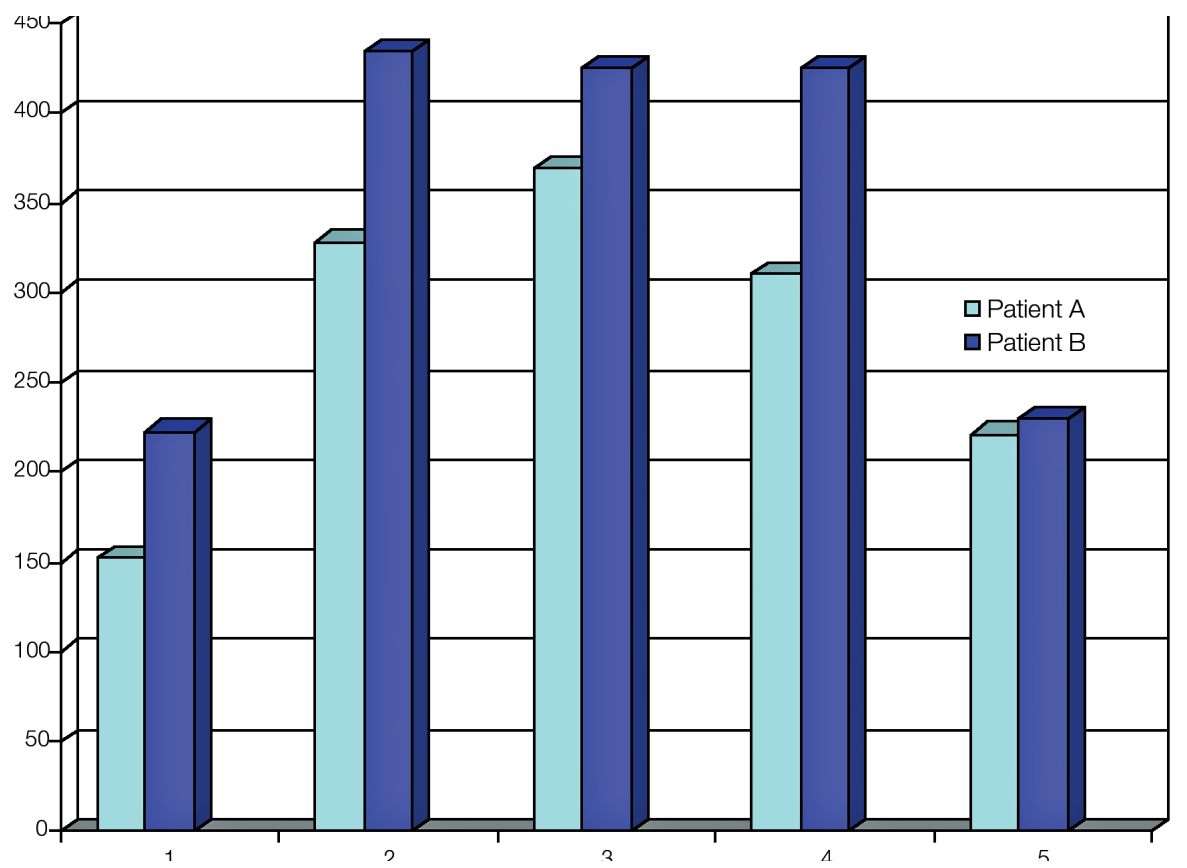

Figure 2 : Coûts globaux des différents modes de prise en charge du patient sous AVK pour l'extraction d'une dent incluse (sauf relais aux HNF avec hospitalisation selon le protocole de Mehra et coll. [2]).

Cost of an included tooth's extraction according to the used protocol; except the Mehra's protocol.

1. Sans modification du traitement AVK

2. Relais avec des HNF sans hospitalisation selon le protocole de Pontchaillou (Rennes)

3. Relais avec des HBPM selon le protocole de Kovacs et coll.

4. Relais avec des HBPM selon le protocole de Pontchaillou (Rennes)

5. Interruption du traitement AVK 
- I'hospitalisation du patient lors d'un relais aux HNF par voie intraveineuse est l'attitude la plus coûteuse.

Cependant, seule une étude prospective pourrait conclure à une éventuelle différence de coût global entre les différentes modalités de prise en charge. Celle-ci serait difficilement réalisable compte tenu du fait que l'arrêt du traitement des AVK est une attitude qui n'est plus recommandée et la mise en place d'un relais héparinique est exceptionnellement nécessaire en chirurgie buccale. L'absence d'archivage des dossiers de patients sous AVK ayant subi une intervention de chirurgie buccale dans notre service n'a pas permis de réaliser une étude rétrospective.

Ces simulations ont dû intégrer de multiples approximations basées sur des moyennes constatées cliniquement :

- le nombre de jours nécessaires pour que le traitement anticoagulant soit de nouveau stable après un relais avec les héparines.

- la quantité de Tissucol ${ }^{\circledR}$ utilisée lors de la réalisation de l'hémostase de niveau 2.

- le nombre d'examens biologiques qui dépend du niveau d'anticoagulation du patient, du nombre de jours nécessaires avant la stabilisation du traitement AVK après relais héparinique, de la physiologie du patient et des habitudes de l'équipe médicale.

\section{CONCLUSION}

Cette étude semble montrer qu'intervenir en chirurgie buccale chez le patient hypocoagulé sans modifier le traitement AVK est l'attitude la moins coûteuse. La SFMBCB recommande ce type de prise en charge pour les interventions potentiellement peu hémorragiques chez le patient dont I'INR est inférieur à 4 et pour les interventions potentiellement hémorragiques chez le patient dont l'INR est inférieur à 3,5 . En effet, elle permet de limiter au maximum le risque de survenue d'accident thromboembolique et, si elle est associée à une hémostase locale de qualité, sans exposer le patient à un risque d'accident hémorragique important. À une époque où les dépenses de santé publique sont au centre des préoccupa- tions dans la société française, il semble important de suivre ces recommandations et de généraliser cette attitude clinique moins onéreuse pour la Sécurité Sociale. Cette étude montre également que lors de la réalisation d'un relais héparinique, l'administration d'HBPM par voie sous-cutanée sans hospitalisation, par une infirmière à domicile, paraît la technique la moins coûteuse. Les différents protocoles de relais avec des HBPM retrouvés dans la littérature sont moins contraignants pour le patient car ils nécessitent moins d'injections journalières et moins d'examens biologiques que lors d'un relais effectué avec les HNF. De plus, la plupart des études montrent que le risque d'effets secondaires est plus faible [7-9]. Ainsi, compte tenu du fait qu'il semble plus sûr, moins contraignant et moins coûteux, le relais avec des HBPM sans hospitalisation, administrées par une infirmière à domicile, semble être le relais à conseiller lors des interventions à risque hémorragique important chez les patients hypocoagulés à risque thromboembolique important. Cette étude montre enfin qu'en plus d'augmenter le risque de survenue d'accidents thromboemboliques, avec l'arrêt du traitement AVK, une intervention de chirurgie buccale est plus onéreuse qu'une intervention sans modification du traitement AVK. Ainsi, comme le stipule les recommandations de la SFMBCB et de la SFC, l'interruption du traitement lors d'une intervention de chirurgie buccale n'est plus une attitude recommandable et raisonnable.

Nous pouvons également remarquer que, quelle que soit l'attitude clinique, la réalisation d'une hémostase locale chez le patient hypo ou anticoagulé nécessite l'utilisation de moyens supplémentaires coûteux pour le praticien ou la structure hospitalière, sans que ceux-ci soient prise en charge par la Sécurité Sociale, car le coût d'un acte de chirurgie buccale comprend également le coût des moyens à mettre en œuvre pour réaliser l'intervention.

II semble donc, au regard de cette étude, que les recommandations de la SFMBCB et de la SFC pour la prise en charge de patients sous AVK en chirurgie buccale aient un rapport coût/sécurité pour le patient largement positif. médecine

buccale

chirurgie

buccale

VOL. $15, \mathrm{~N}^{\circ} 1$ 2009

page 29 


\section{RÉFÉRENCES}

1 - Société Francophone de Médecine Buccale et de Chirurgie Buccale. Prise en charge des patients sous traitement anti-vitamines Ken chirurgie bucco-dentaire. Recommandations. Med Buc Chir Buc 2006 ; 12 : 187 212.

2 - Mehra P, Cottrell DA, Bestgen SC, Booth DF. Management of heparin therapy in the high-risk, chronically anticoagulated, oral surgery patient: a review and a proposed nomogram. J Oral Maxillofac Surg 2000 ; $58:$ 198-202.

3 - Kovacs MJ, Kearon C, Rodger M, Anderson DR, Turpie AG, Bates SM, Desjardins L, Douketis J, Kahn SR, Solymoss S, Wells PS. Single-arm study of bridging therapy with low-molecular-weight heparin for patients at risk of arterial embolism who require temporary interruption of warfarin. Circulation $2004 ; 110$ : 1658-63.

4 - Benoliel R, Leviner E, Katz J, Tzukert A. Dental treatment for the patient on anticoagulant therapy: prothrombin time value - what difference does it make? Oral Surg Oral Med Oral Pathol 1986 ; 62 : 149-51.

médecine buccale chirurgie buccale

VOL. $15, \mathrm{~N}^{\circ} 1$ 2009

page 30
5 - Della Valle A, Sammartino G, Marenzi G, Tia M, Espedito di Lauro A, Ferrari F, Lo Muzio L. Prevention of postoperative bleeding in anticoagulated patients undergoing oral surgery: use of platelet-rich plasma gel. J Ora Maxillofac Surg 2003 ; 61 : 1275-8.
6 - White $\mathrm{RH}$, McKittrick T, Hutchinson R, Twitchell J. Temporary discontinuation of warfarin therapy: changes in the international normalized ratio. Ann Intern Med $1995 ; 122: 40-2$.

7 - Agence Française de Sécurité Sanitaire des Produits de Santé (AFSSAPS). Héparines de bas poids moléculaire. Janvier 2002.

8 - Cestac P, Bagheri H, Lapeyre-Mestre M, Sie P, Fouladi A, Maupas E, Leger P, Fontan B, Massip P, Montastruc JL. Utilisation and safety of low molecular weight heparins: prospective observational study in medical inpatients. Drug Saf 2003 ; 26 : 197-207.

9 - D’Addio VJ, Clagett GP. Commentary. Low-molecularweight heparin as bridging anticoagulation during interruption of warfarin: assessment of a standardized periprocedural anticoagulation regimen. Perspect Vasc Surg Endovasc Ther $2005 ; 17: 176-7$. 